\title{
R-matrix approach at the intersection with the statistical model regime
}

\author{
Thomas Srdinko ${ }^{\mathrm{a}}$ and Helmut Leeb ${ }^{\mathrm{b}}$ \\ TU-Wien, Atominstitut, Wiedner Hauptstrasse 8-10, 1040 Wien, Austria
}

\begin{abstract}
A hybrid R-matrix approach is proposed which combines the pole term decomposition of the R-matrix and a background R-matrix which is based on a coupled-channel pseudo-potential. The pseudopotential is appropriately chosen in order to recover the observables obtained by the statistical model at the energy-onset of its validity. The additional pole terms describe the resonance structure at low energy not attainable in statistical model calculations. Due to its construction the hybrid method allows a smooth transition at the intersection with the statistical model regime. A new R-matrix code based on this hybrid approach was constructed. First applications to $\mathrm{n}^{16} \mathrm{O}$ nuclear data between 0.2 and $5.0 \mathrm{MeV}$ are very promising.
\end{abstract}

\section{Introduction}

R-matrix theory, originally proposed by Wigner and Eisenbud [1-3], is an elegant tool to describe resonant behaviour of cross sections without taking recourse to the complexity of the nuclear many-nucleon problem. In nuclear data evaluation for nuclei with mass number $A \geq 20$ R-matrix based approaches have been successfully applied for the description of experimentally measured reaction cross sections at low incident energies which usually exhibit many resolved resonances. At higher energies the measured reaction cross sections show only smooth energy dependences which are fairly well reproduced by statistical model calculations complemented by other bulk models, e.g. precompound or fission models [4]. Thus, evaluated data files of high quality can be generated for these nuclei.

For light nuclei $(A \leq 20)$ these methods yield less satisfactory results. One reason is the lack of microscopic models which provide quantitatively reliable values of reaction cross sections. Another reason is the small level density of the involved compound nuclei which limits the applicability of the statistical model to rather high excitation energies. Because of the conceptual differences between the two approaches the continuous transition between the R-matrix regime and statistical model calculations represents a challenge and is not obvious. In addition one has also to manage requirements related with the extension of unitary R-matrix calculations up rather high energies.

In standard R-matrix codes a parametrization in terms of pole positions and associated widths parameters is used. In order to develop a procedure which guarantees continuity between the resonance regime and statistical model calculations, we must go beyond a phenomenological description and use the R-matrix formalism

\footnotetext{
a e-mail: tsrdinko@ati.ac.at

b e-mail: leeb@kph.tuwien.ac.at
}

as a tool for the solution of coupled-channels [5]. The corresponding theory is briefly sketched in Sect. 2 and implemented in our new R-matrix code which contains besides the standard resonance parameter representation of the R-matrix also a coupled-channel solver accounting for so-called pseudo-potentials, effective interactions depending on the relative coordinates of the relevant channels. The pseudo-potential approach should be sufficiently flexible to provide a fair description of statistical model calculations and simultaneously represents a proper basis for an extended R-matrix description. In Sect. 3 some details of the numerical implementation and first results on $\mathrm{n}-{ }^{16} \mathrm{O}$ cross sections are discussed. A summary and a brief outlook is given in Sect. 4.

\section{Theory basis}

\subsection{Concept of R-matrix}

The key idea of R-matrix theory is the separation of the configuration space in an inner and an outer region. This idea by Wigner and Eisenbud [1-3] was elaborated by many authors, e.g. [5,6]. Assuming that the interaction in the outer region is known (vanishing potential or Coulomb potential) the wave function of the external region in channel $c$ is known up to the corresponding S-matrix element for open and closed channels,

$$
u_{c\left(c_{0}\right)}^{\mathrm{ext}}(r)= \begin{cases}C_{c}\left[I_{c}(k r) \delta_{c c_{0}}-S_{c c_{0}} O_{c}(k r)\right] & (\text { open }) \\ A_{c c_{0}} W_{-\eta, l+\frac{1}{2}}\left(2 \kappa_{c} r\right) & \text { (closed). }\end{cases}
$$

In Eq. (1) $I_{c}$ and $O_{c}$ are incoming and outgoing Coulomb functions, respectively, in case of open channels, $W_{-\eta, l+\frac{1}{2}}$ represents the Whittaker function in case of closed channels, the magnitudes $C_{c}$ and $A_{c c_{0}}$ are normalization constants.

The wave functions of the inner region reflect the effects of nuclear and Coulomb interactions on the 
relative motion and are usually unknown. Therefore the inner wave functions are described by a superposition of basisfunctions $\varphi_{j}(r)[3]$,

$$
u_{c}^{\mathrm{int}}(r)=\sum_{j=1}^{N} c_{c j} \varphi_{j}(r)
$$

The coefficients $c_{c j}$ in Eq. (2) provide the continuity of the logarithmic derivative at a given matching point $r=a$,

$$
u_{c}^{\mathrm{int}}(a)=u_{c\left(c_{0}\right)}^{\mathrm{ext}}(a) \quad u_{c}^{\mathrm{int}^{\prime}}(a)=u_{c\left(c_{0}\right)}^{\mathrm{ext}^{\prime}}(a) .
$$

These conditions lead to the definition of the R-matrix for coupled-channels

$$
u_{c}^{\mathrm{int}}(r)=\sum_{c^{\prime}} \sqrt{\frac{\mu_{c}}{\mu_{c^{\prime}}}} R_{c c^{\prime}}(E)\left[a u_{c^{\prime}}^{\prime}(a)-B_{c} u_{c^{\prime}}(a)\right],
$$

where $\mu_{c}$ represents the reduced mass in channel $c$ and $B_{c}$ is an arbitrary boundary parameter in channel $c$ which does not affect the observables [5]. For our calculations we choose $B_{c}=0$. However, another choice of $B_{c}$ can be made if it is more appropriate.

\subsection{The calculable R-matrix}

In the following we briefly sketch the formalism of the calculable R-matrix following [5]. The starting point is the solution of the radial part of the coupled-channel Schrdinger equation within the inner region $0 \leq r \leq a$

$$
\left[\left(T_{c}+E_{c}-E\right) \delta_{c c^{\prime}}+V_{c c^{\prime}}(r)\right] u_{c^{\prime}\left(c_{0}\right)}(r)=0,
$$

where $E_{c}$ is the threshold in channel $c$ and $V_{c c^{\prime}}$ the pseudopotential. In these first considerations, non-orthogonality terms of the coupled-channel wave functions are neglected.

Due to the restriction of Eq. (5) to the inner region $(0 \leq r \leq a)$ the Hamiltonian is not Hermitian [6]. The hermiticity can be restored by extending the Schrdinger equation with a surface term - the Bloch operator [7] $\mathcal{L}$

$$
\mathcal{L}=\sum_{c}|c\rangle \mathcal{L}_{c}\langle c|
$$

with

$$
\mathcal{L}_{c}=\frac{\hbar^{2}}{2 \mu_{c}} \delta(r-a)\left(\frac{d}{d r}-\frac{B_{c}}{r}\right),
$$

leading to the Bloch-Schrdinger equation

$$
\left.\sum_{c^{\prime}}\left[T_{c}+\mathcal{L}_{c}+E_{c}-E\right) \delta_{c c^{\prime}}+V_{c c^{\prime}}\right] u_{c}^{\mathrm{int}}(r)=\mathcal{L}_{c} u_{c^{\prime}}^{\mathrm{ext}}(r) .
$$

The wave functions of inner and outer region are separated as can be easily seen from Eq. (8).

The multi-channel R-matrix $R_{c c^{\prime}}(E)$ can be written as

$$
R_{c c^{\prime}}(E)=\frac{\hbar^{2}}{2 \sqrt{\mu_{c} \mu_{c^{\prime}}} a} \sum_{i, i^{\prime}=1}^{N} \varphi_{i}(a)\left(C^{-1}\right)_{c i, c^{\prime} i^{\prime}} \varphi_{i^{\prime}}(a)
$$

where $C_{c i, c^{\prime} i^{\prime}}$ are the matrix elements of the Hamiltonian,

$$
C_{c i, c^{\prime} i^{\prime}}=\left\langle\varphi_{i}\left|T_{c}+\mathcal{L}_{c}+E_{c}-E\right| \varphi_{i^{\prime}}\right\rangle \delta_{c c^{\prime}}+\left\langle\varphi_{i}\left|V_{c c^{\prime}}\right| \varphi_{i^{\prime}}\right\rangle .
$$

Because of hermiticity a spectral decomposition of the Rmatrix can be formulated

$$
R_{c c^{\prime}}(E)=\sum_{n} \frac{\gamma_{n c} \gamma_{n c^{\prime}}}{E_{n}-E}
$$

where the real poles $E_{n}$ are the eigenvalues of the matrix $\boldsymbol{C}(E)$ at $E=0$ and the reduced width-amplitudes $\gamma_{n c}$ of a pole $E_{n}$ in channel $c$ are expressed as a function of the corresponding normed eigenvectors $\boldsymbol{v}_{n}$,

$$
\gamma_{n c}=\left(\frac{\hbar^{2}}{2 \mu_{c} a}\right)^{1 / 2} \sum_{i=1}^{N} v_{n, c i} \varphi_{i}(a) .
$$

The dimension of the matrix $\boldsymbol{C}$ is equal to the product of the number of channels by the number of basis functions $N$.

The scattering matrix $\boldsymbol{S}$ can be directly obtained from the R-matrix with

$$
S=Z_{\mathrm{O}}^{-1} Z_{\mathrm{I}}
$$

with the matrix elements of $\boldsymbol{Z}_{\mathrm{O}}$ and $\boldsymbol{Z}_{\mathrm{I}}$ given by

$$
Z_{\mathrm{O} c c^{\prime}}=\left(k_{c^{\prime}} a\right)^{-1 / 2}\left[O_{c}\left(k_{c} a\right) \delta_{c c^{\prime}}-k_{c^{\prime}} a R_{c c^{\prime}} O_{c^{\prime}}^{\prime}\left(k_{c^{\prime}} a\right)\right]
$$

and

$$
Z_{\mathrm{I} c c^{\prime}}=\left(k_{c^{\prime}} a\right)^{-1 / 2}\left[O_{c}\left(k_{c} a\right) \delta_{c c^{\prime}}-k_{c^{\prime}} a R_{c c^{\prime}} I_{c^{\prime}}^{\prime}\left(k_{c^{\prime}} a\right)\right] .
$$

It is important to point out that the dimension of the $\mathrm{R}$-matrix is equal to the number of all channels, whereas the dimension of the scattering matrix is equal to the number of open channels. Hence the dimension of the $\mathrm{R}$-matrix is constant for all energies while the dimension of the scattering matrix might vary with energy.

From the knowledge of the S-matrix it is possible to calculate the scattering amplitude allowing to determine all observables (e.g. [5]).

\subsection{The phenomenological R-matrix}

Following [1-3,5] it becomes obvious that the R-matrix is essentially the inverse logarithmic derivative at the matching radius $a$. The phenomenological approach makes direct use of Eq. (11) adjusting the reduced widths $\gamma_{n c}$ and poles $E_{n}$ to experimental data.

In practice this yields a very good description of experimental cross sections, but cannot provide predictions. Because no information about the microscopic Hamiltonian is considered the physical interpretation is also limited.

\subsection{Hybrid approach and pseudo-potential}

Standard R-matrix codes use the spectral decomposition of Eq. (11) considering the pole positions $E_{n}$ and associated widths $\gamma_{n c}$ as parameters. Due to the lack of a reliable microscopic procedure for the determination of $E_{n}$ and the widths $\gamma_{n c}$ these quantities have to be adjusted in order to reproduce experimental data. In the calculable $\mathrm{R}$-matrix the number of pole terms is large (number of channels $\times$ number of basis functions), but only few poles lead to obvious resonances in the observables, while remaining poles constitute the background interaction. 
Therefore, phenomenological R-matrix approaches use only the minimal number of poles and describe the background with few so-called shadow poles. In addition simplifications are applied which provide a more direct relation to the S-matrix and thus to the observables. In most cases this leads to an excellent agreement with experimental data. However, due to the lack of a microscopic basis predictions and physics interpretations, e.g. identification of true many-body effects, are not possible. The latter is of importance with regard to the transition between the resonance regime and statistical model calculations.

In order to guarantee a smooth transition between the two regimes the observables from the statistical model calculation are considered to be generated by a coupledchannel pseudo-potential $V_{c c^{\prime}}$. Thus the pseudo-potential represents an effective interaction obtained via projection of the microscopic many-body interaction onto channel states. The pseudo-potential $V_{c c^{\prime}}$ is in principle non-local and depends on the relative coordinates of the channels $c$ and $c^{\prime}$. As an effective potential it is frequently assumed to be local [5] which simplifies the coupled-channel calculations. In this work the assumption is even more justified since the statistical model relies on a completely different concept and the pseudo-potential $V_{c c^{\prime}}$ cannot be derived microscopically and provides only an effective description. Nevertheless it can be expected to be a smooth function of $r$ because the channel thresholds $E_{c}$ are already contained in Eq. (8). Hence a parametrized form of the pseudo-potential has to be assumed whose parameters have to be adjusted to reproduce the observables obtained via statistical model calculations.

The determination of the R-matrix starts from solving the Bloch-Schrdinger Eq. (8) with the pseudo-potential included. With proper basis functions $\varphi_{i}(r)$ one obtains from Eq. (10) the matrix $C$ and thus the R-matrix $R_{c c^{\prime}}^{\mathrm{bg}}(E)$ via Eq. (9). In order to describe experimental observables we use $R_{c c^{\prime}}^{\mathrm{bg}}(E)$ as a background R-matrix and add pole terms

$$
R_{c c^{\prime}}(E)=R_{c c^{\prime}}^{\mathrm{bg}}(E)+\sum_{m} \frac{\gamma_{m c} \gamma_{m c^{\prime}}}{E_{m}-E} .
$$

The poles $E_{m}$ and the associated reduced widths $\gamma_{m c}$ have to be adjusted in order to reproduce the resonances of the experimental cross sections which cannot be described within the statistical model. In general it is expected that the $\gamma_{n c}$ are small and the poles $E_{m}$ lie within the energy region of observed resonances. Thus the effect of additional pole terms will be negligible in the statistical model regime. Thus the ansatz Eq. (15) should guarantee a continuous transition between R-matrix and statistical model regimes. Because the background Rmatrix, $R_{c c^{\prime}}^{\mathrm{bg}}(E)$, is optimized for reproducing statistical model calculations. In addition, the additional pole terms will reflect the many-nucleon structure of the compound nucleus, while $R_{c c^{\prime}}^{\mathrm{bg}}(E)$ accounts for bulk contributions.

\section{Implementation and results}

For the application of the hybrid approach a new R-matrix code was developed which includes the standard spectral decomposition of the phenomenological R-matrix as well as the determination of a background R-matrix within the coupled-channel formalism. For the solution of

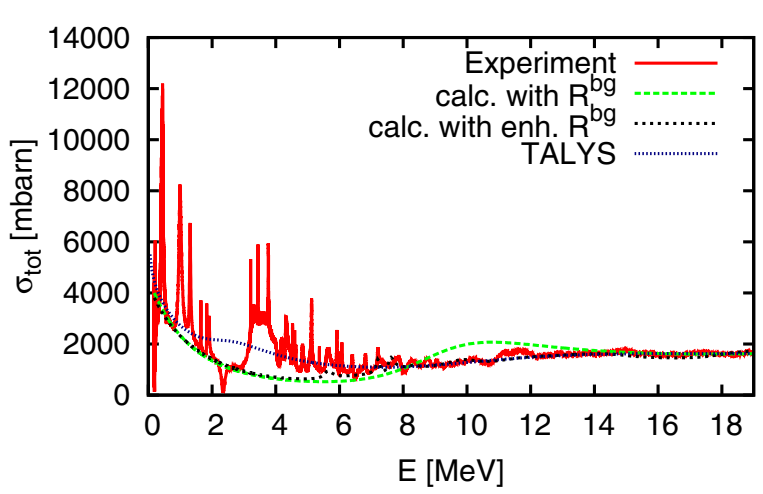

Figure 1. Experimental total cross section in comparison with the cross sections obtained from background R-matrix spectrum, the modified background spectrum and a statistical model TALYS calculation.

the coupled Bloch-Schrdinger equation (8) the Lagrange mesh technique [5] was applied. The use of Lagrange functions for $\varphi_{i}(r)$ simplifies the determination of the matrix $\boldsymbol{C}$ significantly. However, the determination of the eigenvalues $E_{n}$ and eigenvectors $v_{n c}$ is still a bottleneck due to the high number of dimensions (number of channels $\times$ number of basis functions) in matrix $\boldsymbol{C}$. The number of channels is also rising with increasing energy and requiring large scale computing capabilities.

The optimization of the parameters of the hybrid approach $\left(E_{n}, \gamma_{n c}\right.$ and the background potential) is performed by the DAKOTA package [8].

\subsection{Example: Neutron-induced reaction on ${ }^{16} \mathrm{O}$}

The hybrid approach is particularly well suited for light nuclei. For a first test of the hybrid approach we chose neutron-induced reactions on ${ }^{16} \mathrm{O}$ which is a system of great interest at present. As a first guess of the pseudo-potential we use the real part of the Woods-Saxon shaped volume term taking the parameters from the RIPL3 library [9]. Slight optimization of the Woods-Saxon parameters already reproduced some of the basic cross section structure as can be seen in Fig. 1. An imperfection shows up at about $10 \mathrm{MeV}$ where the cross section has a slight bump.

All poles with different $J^{\pi}$ but the same $l$ are identical because the only angular momentum dependence of the Hamiltonian is in the centrifugal term of the kinetic energy operator. Varying the positions and widths of the poles in the relevant region from $0-10 \mathrm{MeV}$, as illustrated in Fig. 2, the bump at $10 \mathrm{MeV}$ can be healed. This indicates the necessity of some $J$-dependence of the background pseudo-potential.

In order to show the transition to the statistical model regime we performed calculations with the TALYS code [10]. As one can see in Fig. 1 our background Rmatrix calculation coincides nicely with the experimental data and the statistical model calculation beyond $14 \mathrm{MeV}$.

The resonance structure of the cross section can be reproduced by adding pole terms to the background R-matrix (Eq. (15)), displayed in Fig. 3. Within the range from $0.2-5 \mathrm{MeV}$ the resonance structure is nicely reproduced while a physically reasonable matching radius of $a=7 \mathrm{fm}$ is kept. 


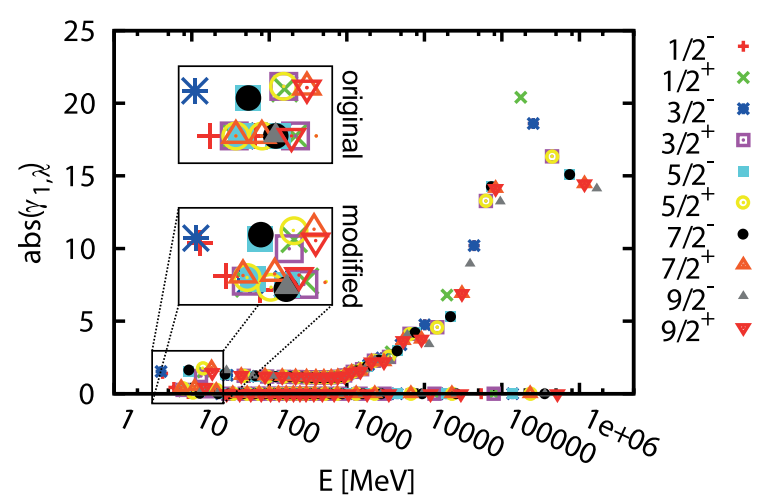

Figure 2. Positions of background poles and the absolute value of their corresponding widths for various partial waves for the original spectrum obtained from pseudo-potential and the modified spectrum.

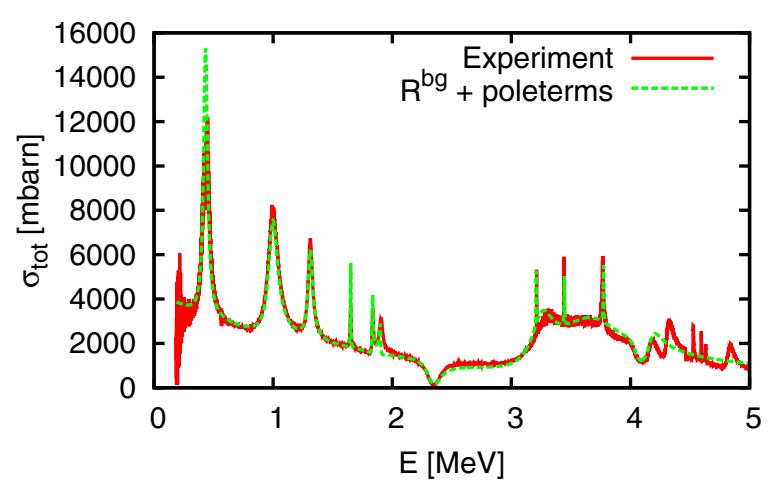

Figure 3. Experimental total cross section in comparison with cross section obtained by the hybrid R-matrix approach (background spectrum + additional pole terms).

\section{Summary and outlook}

The presented hybrid approach which combines a background spectrum obtained from a coupled-channel calculation with additional pole terms seems very promising. The background potential was able to reproduce the basic structure of the total cross section at energies beyond $13 \mathrm{MeV}$ using a physically reasonable matching radius of $7 \mathrm{fm}$. With additional pole terms the resonance structure from $0.2-5 \mathrm{MeV}$ could be reproduced. Yet the fit is not perfect and is more to be seen as a proof of concept. Furthermore when going to higher energies more channels have to be included leading to more parameters. Interference effects and nonlinearity effects raise the demand for versatile fitting routines.
The proper parametrization of the background potential has shown to be crucial for the shape of the basic cross section structure. Our studies clearly show the necessity of a J-dependent background potential. The required modifications in the background spectrum were small and therefore the corresponding effect on the pseudo-potential will be extracted via perturbative methods.

The contributions of the $(n, \gamma)$ channel were neglible. In the transition region starting at about $13 \mathrm{MeV}, 3$-body reactions will play an important role. The proper inclusion of $(\mathrm{n}, \gamma)$ and 3-body processes in our R-matrix code is currently work in progress.

Work partly supported by Fusion for Energy via the specific grant F4E-FPA-168.02 and the Austrian Academy of Sciences via the KKK matching grant MG 2014-4. The views and opinions expressed herein do not reflect necessarily those of the European Commission.

\section{References}

[1] E.P. Wigner, Phys. Rev. 70, 15 (1946)

[2] E.P. Wigner, Phys. Rev. 70, 606 (1946)

[3] E.P. Wigner, L. Eisenbud, Phys. Rev. 72, 29 (1947)

[4] A. Koning, E. Bauge, C. Dean, E. Dupont, U. Fischer, R. Forrest, R. Jacqmin, H. Leeb, M. Kellett, R. Mills et al., Journal of the Korean Physical Society 59/2, 1057 (2011)

[5] P. Descouvemont, D. Baye, Reports on Progress in Physics 73, 036301 (2010)

[6] A.M. Lane, R.G. Thomas, Rev. Mod. Phys. 30, 257 (1958)

[7] C. Bloch, Nucl. Phys. 4, 503 (1957)

[8] B. Adams, L. Bauman, W. Bohnhoff, K. Dalbey, M. Ebeida, J. Eddy, M. Eldred, P. Hough, K. Hu, J. Jakeman et al., DAKOTA, A Multilevel Parallel Object-Oriented Framework for Design Optimization, Parameter Estimation, Uncertainty Quantification, and Sensitivity Analysis: Version 5.4 User's Manual, Sandia Technical Report SAND2010-2183 (2009), updated April (2013)

[9] R. Capote, M. Herman, P. Obložinský, P. Young, S. Goriely, T. Belgya, A. Ignatyuk, A. Koning, S. Hilaire, V. Plujko et al., Nuclear Data Sheets 110, 3107 (2009)

[10] A. Koning, S. Hilaire, S. Goriely, TALYS - A nuclear reaction program (2015), http: //www.talys .eu/ home/ 